\title{
Atenção à hipertensão e diabetes na Amazônia: a percepção de usuários da região metropolitana de Belém
}

\author{
Attention to hypertension and diabetes in the Amazon: the perception of users from the \\ metropolitan region of Belem
}
Atención a la hipertensión y diabetes en la Amazonía: la percepción de los usuarios de la región metropolitana de Belém

Fábio da Costa Ferreira ${ }^{1 *}$, Marcos Valério Santos da Silva.

\section{RESUMO}

Objetivo: Compreender a percepção dos usuários acerca da assistência a hipertensão e diabetes no Sistema Único de Saúde (SUS) na região metropolitana de Belém do Pará. Métodos: Estudo descritivo, do tipo estudo de caso, com abordagem qualitativa. As fontes de evidências foram documentais constituídas por elementos normativos sobre atenção a hipertensão e diabetes, artigos científicos, livros e entrevista com perguntas abertas coletada por meio de gripo focal com usuários do Sistema Único de Saúde. Resultados: Foi realizada 2 focalizações com 6 usuários cada reunião. A partir da abordagem qualitativa, realizou-se a análise de conteúdo das focalizações, emergindo 4 categorias, suscitando em proposições a atenção a hipertensão e diabetes. Conclusão: A pesquisa foi realizada em apenas uma Unidade Básica de Saúde (UBS), o que restringe a da análise, contudo compreende-se que os desafios da atenção a hipertensão e diabetes na região metropolitana de Belém do Pará, perpassam pelo conhecimento dos profissionais, gestores e pela vivência dos usuários do Sistema Único de Saúde, ou seja a eficácia decorrem pela habilidade da gestão em organizar os serviços com o foco no atendimento das necessidades da população, respeitando as características geográficas e sociais da comunidade.

Palavras-chave: Hipertensão arterial, Diabetes mellitus, Assistência em saúde.

\begin{abstract}
Objective: To understand the users' perception about the assistance to hypertension and diabetes in the Unified Health System (SUS) in the metropolitan region of Belém do Pará. Methods: Descriptive study, of the case study type, with a qualitative approach. The sources of evidence were documentary constituted by normative elements on attention to hypertension and diabetes, scientific articles, books and an interview with open questions collected through a focal group with users of the Unified Health System. Results: 2 focuses were carried out with 6 users each meeting. From the qualitative approach, the content analysis of the focus was carried out, emerging 4 categories, raising in proposals the attention to hypertension and diabetes. Conclusion: The research was carried out in only one Basic Health Unit (UBS), which restricts the analysis, however it is understood that the challenges of attention to hypertension and diabetes in the metropolitan region of Belém do Pará, permeate the knowledge of professionals, managers and the experience of the users of the Unified Health System, that is, efficiency is due to the ability of management to organize services with a focus on meeting the needs of the population, respecting the geographical and social characteristics of the community.
\end{abstract}

Keywords: Arterial hypertension, Diabetes mellitus, Health care.

${ }^{1}$ Universidade Federal do Pará (UFPA), Belém - PA. *E-mail: ferreirafabio582@gmail.com 


\section{RESUMEN}

Objetivo: Comprender la percepción de los usuarios sobre la atención a la hipertensión y diabetes en el Sistema Único de Salud (SUS) en la región metropolitana de Belém do Pará. Métodos: Estudio descriptivo, tipo estudio de caso, con abordaje cualitativo. Las fuentes de evidencia fueron documentales constituidas por elementos normativos sobre atención a la hipertensión y diabetes, artículos científicos, libros y una entrevista con preguntas abiertas recolectadas a través de un grupo focal con usuarios del Sistema Único de Salud. Resultados: Se realizaron 2 focos con 6 usuarios cada encuentro. Desde el abordaje cualitativo, se realizó el análisis de contenido del foco, emergiendo 4 categorías, planteando en propuestas la atención a la hipertensión y diabetes. Conclusión: La investigación se realizó en una única Unidad Básica de Salud (UBS), lo que restringe el análisis, sin embargo se entiende que los desafíos de la atención a la hipertensión y diabetes en la región metropolitana de Belém do Pará, permean el conocimiento de los profesionales, gestores y la experiencia de los usuarios del Sistema Único de Salud, es decir, la eficiencia se debe a la capacidad de la gestión para organizar los servicios con un enfoque en la satisfacción de las necesidades de la población, respetando las características geográficas y sociales de la comunidad.

Palabras clave: Hipertensión arterial, Diabetes mellitus, Cuidado de la salud.

\section{INTRODUÇÃO}

Nas últimas décadas, a expectativa de vida dos brasileiros vem aumentando e consequentemente a população de idosos também. Diante deste cenário, houve uma importante mudança no perfil da morbidade e mortalidade da população brasileira, com aumento dos agravos e óbitos causados por doenças crônico não transmissíveis e causas externas (BRASIL, 2013ab). As doenças cardiovasculares são as causas mais comuns de morbidade e mortalidade em todo o mundo e, entre os fatores de risco para doença cardiovascular, encontram-se a hipertensão arterial sistêmica (HAS) e o diabetes mellitus (DM), fatores simultâneos e autônomos (WHO, 2009).

A HAS é uma condição clinica multifatorial caracterizada por níveis pressóricos elevados e sustentados da pressão arterial. No Brasil, 21,4\% da população de 18 anos e mais referem ter o diagnóstico de HAS; já na região norte, a prevalência em indivíduos acima de 18 anos apresentou 14,5\%. O DM é um transtorno metabólico caracterizado por hiperglicemia e distúrbios do metabolismo de macro nutrientes como carboidratos, proteínas e gorduras. A prevalência na população brasileira foi estimada em $6,2 \%$ de indivíduos acima de 18 anos, já na região norte foi estimada em 4,3\% (BRASIL, 2013ab).

O controle da HAS perpassa pela redução de danos aos órgãos-alvo. E o controle metabólico e os cuidados curativos são capazes de prevenir ou desacelerar as complicações do DM, resultando em melhor qualidade de vida do portador. Para o controle de ambas as patologias, são necessárias medidas que envolvam mudanças no estilo de vida do indivíduo (BRASIL, 2013a).

Dentro da Atenção Básica (AB) no Estratégia Saúde da Família (ESF), foi apresentado o Plano de Reorganização da atenção à Hipertensão Arterial e ao Diabetes Mellitus, o qual constitui-se em uma ferramenta de controle e acompanhamento dos hipertensos e/ou diabéticos, trabalhando a promoção e proteção da saúde, na prevenção de agravos, no diagnóstico, no tratamento, na reabilitação e manutenção da saúde. Para tanto, utilizam-se de fichas com vistas a realizar o cadastro e o acompanhamento dos usuários no sistema de Hipertensão e Diabetes (HIPERDIA) (substituído pelo e-SUS), bem como fazer avaliação de risco entre os pacientes cadastrados (BRASIL, 2001).

Este estudo tem relevância no âmbito da saúde coletiva por tratar de um problema que acomete a população brasileira e mundial, que são as doenças do aparelho circulatório. Das quais, a consequência é a alta taxa de morbimortalidade e hospitalizações relacionadas aos agravos cardiovasculares e renais como infarto agudo do miocárdio (IAM), acidente vascular encefálico (AVE), insuficiência renal crônica (IRC), dentre outros (CAMPOLINA AS, et al., 2013; BONOW RO, et al., 2002).

Nesse cenário, o Plano de Reorganização da atenção à Hipertensão Arterial e ao Diabetes Mellitus coordenado e organizado pela $A B$ e executado pelas equipes do programa ESF tem um papel fundamental 
por trazer um impacto na redução dessas doenças por meio do cadastramento, acompanhamento e no controle dos agravos utilizando um tratamento fundamentado na promoção e proteção à saúde e prevenção dos agravos por meio da ferramenta educação em saúde que proporciona autonomia e autorresponsabilidade do portador de HAS e/ou DM. Além disso, utilizam no tratamento, fármacos que controlam os fatores de riscos (PESSANHA CJS, 2020).

Evidentemente na região norte, há uma escassez de estudos voltados a essa temática, sobretudo a pesquisa apontará achados que poderão fundamentar a tomada de decisão à luz de avaliações sistemáticas e evidências comprovadas buscando aperfeiçoamento e consequente melhoria do desempenho da assistência no acompanhamento da HAS e/ou DM na AB, bem como oferecer dados relacionados aos seus principais avanços e entraves (fragilidades) no controle da hipertensão e diabetes do programa $E S F$ da $A B$ (STOPA SR, et al., 2013; TRAVASSOS M, 2004).

Diante disso, o objetivo geral deste estudo foi compreender a percepção dos usuários acerca da assistência a hipertensão e diabetes no SUS na região metropolitana de Belém Pará.

\section{MÉTODOS}

Trata-se de um estudo exploratório, descritivo e com abordagem qualitativa, sobre a percepção dos usuários acerca da assistência a hipertensão e diabetes na $A B$ na região metropolitana de Belém-PA.

As focalizações ocorreram em duas reuniões em datas distintas nos meses de agosto e setembro de 2020 , com 1 grupo de indivíduos, usuários maiores de 18 anos, cadastrados e acompanhados no mínimo por 6 meses no programa de controle e acompanhamento de hipertensão e diabetes, ativos acompanhados que participaram de ações em saúde mensalmente e por fim que aceitaram participar do estudo e assinaram o Termo de Consentimento Livre e Esclarecido (TCLE). A focalização foi norteada por um roteiro elaborado em concordância com os objetivos da pesquisa, foram gravadas, transcritas na íntegra e armazenada em arquivo de pesquisa.

O grupo foi composto de usuários escolhidos aleatoriamente e que estavam cadastrados e acompanhados por uma equipe ESF da região metropolitana de Belém, totalizando o número de 12 usuários.

Segundo Teixeira E (2009), a abordagem qualitativa possui um espaço reduzido entre o investigador e seu objeto de estudo, possibilitando uma descrição e interpretação dos dados com mais riqueza de detalhes. As vivências pessoais do investigador são elementos de extrema relevância na análise e compreensão do fenômeno pesquisado.

Para a abordagem qualitativa, optou-se pela técnica de análise de conteúdo, a qual organiza-se em três etapas, a saber: pré-análise, exploração do material, e tratamento dos resultados (BARDIN L, 2016). Nesta pesquisa na pré-análise, foram realizadas a leitura flutuante, organização do material, a formulação de hipóteses e a preparação para análise. $\mathrm{Na}$ etapa de exploração, as falas transcritas foram analisadas e organizadas em recortes que se aproximavam quanto ao tema e escolheu-se por agrupar esses dados, formulando assim categorias que transformaram nos temas específicos de estudo. Após a etapa de exploração, emergiram 4 categorias, que foram formulados de acordo com o roteiro de focalização e dos objetivos da pesquisa, são eles: 1) acesso aos serviços de saúde; 2) consultas na unidade de saúde; 3) acolhimento; e; 4) visita domiciliar.

A pesquisa foi realizada seguindo as disposições da resolução 466/12, do conselho nacional de saúde, que trata de pesquisas envolvendo seres humanos. Foi explicado aos participantes os objetivos e os possíveis riscos e benefícios da pesquisa. Após concordância dos usuários ocorreu a assinatura do TCLE, para então serem iniciadas as focalizações.

No que se refere aos riscos, esses foram mínimos, decorrente, porventura, de algum leve desconforto durante as focalizações, os quais foram rapidamente minimizados e resolvidos. Já os benefícios, o estudo contribuiu para a absorção de conhecimento, entendimento na área e ficando à disposição da comunidade cientifica e da sociedade civil, contudo percebe-se carência de maior volume literário sobre a temática. 
Este estudo foi submetido e aprovado pelo Comitê de Ética em Pesquisa (CEP) Envolvendo Seres Humanos da Universidade Federal do Pará (UFPA) em 13/4/2020, conforme CAAE: 28979819.9.0000.0018 e número de parecer: 3.968.535.

\section{RESULTADOS E DISCUSSÃO}

Foram realizadas duas focalizações com 6 participantes cada, totalizando 12 usuários, sendo norteada pelo roteiro elaborado com três perguntas abertas. A partir da análise de conteúdo do grupo focal, foram formuladas as categorias para discussão (Quadro 1).

Quadro 1- Categorias da análise de conteúdo.

\begin{tabular}{|c|}
\hline CATEGORIAS \\
\hline 1. Acesso aos serviços de saúde \\
\hline 2. Consultas na unidade de saúde \\
\hline 3. Acolhimento \\
\hline 4. Visita domiciliar \\
\hline
\end{tabular}

Fonte: Ferreira FC e Silva MVS, 2021.

\section{Acesso aos serviços de saúde}

A percepção do grau de satisfação do usuário está relacionada diretamente com a gestão eficiente, sendo essa gestão responsável em avaliar o serviço no intuito de tomar decisões estratégicas e operacionais que são relevantes para a melhoria da qualidade dos serviços (SILVA JUNIOR G e DIAS E, 2016). Os achados do atual estudo relacionam o acesso aos serviços de saúde com organização do serviço, com sistemas operacionais de distribuição de fichas e uso do ACS com facilitador do usuário ao serviço.

Evidencia-se nas falas dos usuários a dificuldade em entender como se dá o processo de trabalho da equipe de assistência da unidade, implicando em uma possível falta de orientação ou de um modelo assistencial visível a eles. Portanto, percebe-se por meio das falas dos usuários sentimentos de insatisfação e até mesmo desconhecimento dos usuários diante da organização do serviço.

"[...] aí você fica lá esperando, aí não tem médico, aí eles marcam você para o outro dia, mandam você de um lugar para o outro... eu faço tratamento aí porque não tenho condições para ir em outro lugar, devia ser uma coisa mais organizada, não tem ninguém para orientar, você devia chegar e ser logo atendido, não sei quem é o gestor de um posto desses aí, porque se eu fosse gestor eu ia fazer uma coisa exclusiva para os hipertensos e diabéticos para serem atendidos com educação. Porque você sabe quem é hipertenso e diabético quando altera a pressão e a glicose, você corre para onde? Para o posto de saúde! Eu nem vou mais nesse posto e vou ali no outro posto, que por incrível que pareça eu sou bem atendido, quer dizer lá já é centro, lá está tudo informatizado, então era isso que devia o gestor prestar mais atenção nisso aí, era isso" (U7).

"[...] o atendimento da equipe do postinho é muito desorganizado, muito ruim" (12).

No trabalho de Faria HP, et al. (2010), ressaltam que, responsabilizar-se com as expectativas e necessidades de pessoas, famílias ou até mesmo de comunidades exige, dentre muitas coisas, instrumentos, modelos, fluxos assistenciais visíveis e acessíveis que possam orientar as intervenções, cuidados e avaliações adequadas de um serviço assistencial.

Essa forma de promover a acessibilidade por meio da distribuição de fichas demostra pouca ou nenhuma eficácia, devido à demanda que é grande, consequentemente formando filas, obrigando os usuários chegarem o mais cedo possível, essa distribuição das fichas não leva em consideração a localização da unidade e o perigo, já que a comunidade tem histórico de violência, além das filas não serem nada confortáveis para os usuários, já que a espera é do lado de fora da unidade. 
"[...] primeiro que a gente ir de madrugada para lá, ficar a madrugada todinha lá na fila para nem ser atendido" (U7).

"[...] tenho que pegar ficha para guardar um lugar na fila para atendimento, não consigo ir de madrugada como outras pessoas falaram para pegar ficha, o bairro é muito perigoso" (U9).

"[...] mas consulta eu não faço não porque tem que ir de madrugada para fila e o meu esposo não quer ir comigo porque ele trabalha de manhã, eu tenho medo, é perigoso" (U10).

Esses achados do atual estudo corroboram com os achados de outros, realizados em outras regiões do Brasil, onde o modelo tradicional de organização na recepção permanece, e a entrega das fichas e da marcação no balcão, acrescido a esse contexto, tem-se a demanda muito grande que são agravadas pelo tradicional "dormir na fila" em madrugadas frias e medo da violência presente nas regiões metropolitanas do país. Portanto, as filas e fichas continuam sendo motivo de reclamações e insatisfação dos usuários (SOUZA ECF, et al., 2008).

Compreende-se por meio dos relatos dos usuários que o ACS é o mediador mais efetivo, para a promoção de acessibilidade. Os achados sugerem que a relação do ACS e os usuários é muito boa, o que contribui e muito para o acesso aos serviços. Como exemplificado nas falas.

"[...] o ACS, ele leva, marca, ele falou dona U5 está marcada a sua consulta, aí eu fui com o médico" (U5).

"[...] aí a menina (ACS) é muito boa para mim, ela marca a consulta para mim" (U6).

"[...] quem me ajuda é a menina (ACS) porque eu não conheço a ACS da minha área, ela atende muito bem a gente, aí eu me apego a ela quando eu preciso de consulta de médico" (U8).

Os relatos expostos acima corroboram com o estudo de Barralhas M e Pereira MA (2013), onde a contribuição do ACS de chamar a responsabilidade da assistência para si, se reflete quando este faz o contato do usuário com a UBS. Isto é, o ACS se coloca como facilitador do acesso, agendando as consultas, exames, facilitando o recebimento das medicações, dentre outros serviços. Assim, se sentido responsável na efetivação do direito do usuário a assistência.

\section{Consulta na unidade de saúde}

Percebe-se que está muito confuso a forma de marcação de consulta da equipe pois os usuários não conseguem visualizar o fluxograma de agendamento, seja de consultas agendadas ou as espontâneas. Como evidenciado nas falas dos usuários a seguir.

"[... ] a dificuldade que a gente tem é esse aí, porque de primeiro a gente consultava quando a gente saia do consultório já passava lá no arquivo para marcar a consulta de retorno ou para a enfermagem ou para o médico, agora não" (U1).

"[...] acho que a maior parte do pessoal faz acompanhamento lá no posto, tinha uma consulta e eles marcavam logo a nossa volta ou era com a enfermeira ou era com o médico e isso nos facilitava bastante" (U2).

"[...] de primeiro era só uma vez na semana, só na sexta a marcação de consulta. Então acho que não era para o diabético e hipertensos esperar tudo isso, não era para eles fazerem isso, não tem uma vaga, tenho que esperar 8 dias para poder marcar" (U3).

Os achados do atual estudo corroboram com o trabalho de Marinho NBP, et al. (2018) e Viegas APB, et al. (2015), que afirmam, a insatisfação com a forma de marcação de consultas e de exames da unidade, demostra que não há um processo de agendamento, ou qualquer outro tipo de sistema de marcação das consultas e exames, sendo utilizada apenas o sistema de distribuição de fichas, que nem sempre os usuários conseguem marcar as consultas o que acarreta um certo descontentamento por parte dos usuários.

Para as consultas medicas, percebe-se dificuldades em vários aspectos como atenção, procedimentos de aferição, exames e receitas. 
"[...] tinha uma dra. Cubana, ela consultava, passava exame, fazia receita, media nossa pressão, a nossa diabetes, ela foi embora e agora para gente conseguir amostrar esse resultado? É muito difícil! [...] outra dificuldade é para fazer exames porque não faço a consulta não tenho a receita, faz muito tempo que não faço nenhum exame de sangue. Quando conseguimos uma consulta tem tanta gente, esperamos tanto, demora demais, e não tem onde ficar aguardando fica todo mundo em pé, quando a gente entra o médico não tira nossa pressão" (U9).

"[...] de primeiro a gente conseguia se consultar com o doutor, mas ele fica de cabeça baixa e escrevia uma receita e pronto acabava a consulta, as vezes estava com dor de cabeça, mas não tiravam minha pressão, acho que isso acontecia porque tinha muita gente lá fora para ele atender" (U12).

Esses achados corroboram com o estudo de Carvalho Filha FSS, et al. (2014), afirmam que a insatisfação do usuário está principalmente na falta de atenção do médico, acrescido ao tempo reduzido de atendimento por consequência de uma demanda grande e reprimida. Além disso, ainda acontece a não verificação da pressão e glicemia, e mais a receita que não é atendida na farmácia da unidade por falta de medicamentos. Todos esses fatores contribuem para a insatisfação do usuário com as consultas médicas e, consequentemente, com a baixa ou até mesmo com a não adesão ao tratamento, já que os usuários residem em bairro carente e periférico, onde na sua grande maioria possui renda mínima com dificuldade para aquisição dos medicamentos.

\section{Acolhimento}

Percebe-se que a maioria dos usuários que participaram da focalização demostra uma extrema insatisfação com a forma que são recebidos na unidade. Assim como está ilustrado em algumas falas abaixo.

\section{"[...] para mim é péssimo o atendimento de lá" (U1).}

"[...] a respeito do que a dona U5 falou desse posto aí, são muito ignorantes, você é maltratado, entendeu? A gente está ali, não está de graça, não pensa que aquilo é de graça, a gente paga nosso imposto... eu fui uma vez aí e fui mal atendido...você é logo mal atendido na entrada, na recepção, entendeu? ... A pessoa está ali trabalhando todo mal-humorado, parece que está fazendo tudo de mal gosto, se não está satisfeito dá vaga para outro. Os postos de saúde em geral não só o daqui, onde você vai, você é maltratado principalmente aqui nesse posto... e acabei vindo embora sem atendimento porque a mulher é ignorante de pai e mãe" (U7).

Esses achados diferem dos encontrados por outro estudo realizado em Fortaleza, no Ceará, Lima LL, (2013), onde constatou-se a formação de vínculo entre a equipe e os usuários como recomendado por documentos do Ministério da Saúde, tendo como consequência a satisfação pelo atendimento recebido. Contudo, o atual estudo apresentou resultados diferentes, onde dez dos doze usuários apontaram que a relação entre equipe e usuários era hostil, caracterizando um vínculo desarmônico que tem como consequência uma baixa resolutividade das necessidades dos usuários; portanto, a sugestão neste caso é seguir as recomendações do Ministério da Saúde para a organização do serviço e utilizar a educação permanente para desenvolver na equipe a habilidade de reconhecer a necessidade e dá seguimento no atendimento com o foco na resolução da demanda do processo saúde-doença do usuário.

\section{Visita domiciliar}

Constata-se que o ACS cumpre de forma satisfatória a função de ser o contato da comunidade com a equipe de saúde, de desenvolver trabalhos educativos reforçando a promoção à saúde e a prevenção de doenças. Contudo, refletindo tais atribuições, compreende-se que os usuários que participaram do presente estudo não conhecem todas as funções de um ACS, limitam as funções do ACS a marcação de consulta/exames e fornecimento de medicamentos.

"[...] a ACS aqui da nossa comunidade, que tem se esforçado muito para nos ajudar, mas a gente sabe que isso não depende somente dela, ela faz o trabalho dela, entendeu?"(U4). 
"[...] graça a deus o ACS que vem na minha casa, ele pergunta se eu preciso de alguma coisa, se preciso de consulta, ele leva, marca, ele fala dona U5 está marcada sua consulta... então é isso que precisamos, é de pessoas vindo na comunidade, como os ACS, eles ajudam muito" (U5).

Os achados do presente estudo diferem de um estudo realizado em Minas Gerais, Braga GMAM (2016), onde $62 \%$ dos entrevistados referem conhecer as atribuições dos ACS e que $40 \%$ relacionam este como elo de integração da comunidade com a equipe de saúde da UBS. Já com relação às atribuições do ACS, o atual estudo corrobora com o preconizado pelo MS, onde o ACS tem como atribuições o cadastramento, onde coleta as informações de cada membro da família, mapeia a comunidade no intuito de contribuir com planejamento da equipe, identifica as áreas de risco da comunidade, realiza as visitas domiciliares, além de ações coletivas com objetivo de promover à saúde e prevenir os agravos à saúde.

Observa-se por meio das falas dos usuários, a dificuldade de inclusão dos profissionais da equipe nesta atividade, que aparenta ainda se centralizar no ACS. Isso porque a Visita Domiciliar (VD) dos profissionais médicos e enfermeiros são ocasionais, irregulares e imprevistas. Prova disso é a percepção dos usuários quanto à VD ser sentida como algo inesperado, imprevisível e algumas vezes repentino, ou seja, falta de agendamento das visitas.

"[...] ah tem outra coisa só quem atende a gente em casa é o ACS, nunca veio um médico na minha casa" (U9).

"[...] verdade é que só o ACS nos visita, nunca recebi um doutor em casa, acho que uma vez que foi um enfermeiro me perguntou se eu tomava os remédios respondi nem tem remédio no posto, quando tenho dinheiro eu compro, quando não tenho fico sem tomar os remédios" (U11).

"[...] e o doutor nunca vem em casa, uma vez veio uma enfermeira, mas depois nunca mais veio, sempre quem vem visitar é o ACS" (U12).

Esses achados do presente estudo corroboram com um estudo realizado em Nova Iguaçu, no Rio de Janeiro, Cunha MS e Sa MC (2013), afirmam que apesar da VD de médicos e enfermeiros funcionar como um instrumento altamente eficaz no diagnóstico, no planejamento das ações de saúde e na reorientação das práticas assistenciais, ainda esbarra em entraves para seu fortalecimento, principalmente por necessitar de grande disponibilidade por parte dos profissionais de saúde. Outrossim, cabe a equipe multiprofissional planejar e implantar um cronograma de agendamento a partir das disponibilidades dos médicos e enfermeiros. Somente assim, a VD alcançará seu objetivo que é a atenção de saúde aos usuários hipertensos e/ou diabéticos.

\section{CONCLUSÃO}

Considerando os achados desse estudo percebe-se que a assistência prestada aos portadores de HAS e DM, envolvem uma serie de aspectos vivenciados pelos usuários. Assim, pode-se apontar que a percepção dos usuários sobre a temática, norteia suas expectativas quanto as dificuldades apresentadas no acesso, nas consultas, no acolhimento e na visita domiciliar e essa percepção é produto das suas bases históricas, culturais e sociais, de acordo com suas concepções de mundo, assim as dificuldades apresentadas pelos usuários reflete a insatisfação quanto a atenção a HAS e DM recebida por eles. Logo, a eficácia da assistência perpassa pela habilidade da gestão em organizar os serviços com o foco no atendimento das necessidades da população, respeitando as características geográfica e social da comunidade.

\section{REFERÊNCIAS}

1. BARALHAS M, PEREIRA MAO. Pratica diária dos agentes comunitários de saúde: dificuldades e limitações da assistência. Rev. Bras. Enferm, 2013; 66(3): 358-365.

2. BARDIN L. Análise de conteúdo. 1ed. São Paulo: Almedina, 2011.

3. BONOW RO, et al. Prevention conference VI. Diabetes and cardiovascular disease. Writing group V: management of cardiovascular-renal complications. Circulation 2002; 105(18): e159-164.

4. BRAGA GMAM, et al. Percepção do trabalho do agente comunitário de saúde pelos usuários atendidos nas unidades básicas de saúde da família de Viçosa, MG: Tarefas realizadas e normas prescritas. Revista Brasileira de Economia Doméstica, 2016; 27(1): 79-95. 
5. BRASIL, Ministério da saúde. Secretaria de Atenção à Saúde. Departamento de Atenção Básica. Estratégia para o cuidado da pessoa com doença crônica: hipertensão arterial sistêmica. Brasília: Ministério da Saúde, 2013a. Disponível em: bvsms.saude.gov.br. Acessado em: 22 de agosto de 2019.

6. BRASIL, Ministério da saúde. Secretaria de Atenção à Saúde. Departamento de Atenção Básica. Estratégia para o cuidado da pessoa com doença crônica: diabetes mellitus. Brasília: Ministério da Saúde, 2013b. Disponível em: bvsms.saude.gov.br. Acessado em: 22 de agosto de 2019.

7. BRASIL. Ministério da Saúde. Plano de reorganização da atenção à hipertensão arterial e ao diabetes mellitus. Brasília: Ministério da Saúde, 2001. Disponível em: bvsms.saude.gov.br. Acessado em: 22 de agosto de 2019.

8. CAMPOLINA AS, et al. A transição de saúde e as mudanças na expectativa de vida saudável da população idosa: possíveis impactos da prevenção de doenças crônicas. Cad. Saúde Pública, 2013; 29(6): 1217-1229.

9. CARVALHO FILHA FSS, et al. Avaliação do controle de hipertensão e diabetes na atenção básica: perspectiva de profissionais e usuários. Saúde Debate, 2014; 38(esp.): 265-278.

10. CUNHA MS, SÁ MC. A visita domiciliar na estratégia de saúde da família: os desafios de se mover no território. Interface Comunicação, Saúde e Educação, 2013; 17(44): 61-73.

11. FARIA HP. Modelo assistencial e atenção básica a saúde. 2 ed. Belo Horizonte: Coopmed, 2010; 68p.

12. LIMA LL, et al. Produção do cuidado a pessoas com hipertensão arterial: acolhimento, vínculo e corresponsabilização. Rev. Bras. Enferm, 2013; 66(4): 514-522.

13. MARINHO NBP, et. al. Evaluation of the satisfaction of users of a servisse specialized in diabetes mellitus. Rev. Bras. Enferm, 2018; 71(supl.1): 599-606.

14. PESSANHA CJS. O contexto da saúde brasileira e o enfrentamento ao avanço do diabetes mellitus no Brasil: a implantação do plano de reorganização da atenção a hipertensão arterial e ao diabetes mellitus (PRHADM). Mundo livre: Revista multidisciplinar, 2020; 6(2): 283-305.

15. SILVA JUNIOR GB, DIAS ER. Avaliação da satisfação dos usuários de um serviço de saúde público-privado no nordeste do Brasil e a judicialização da saúde. Rev. Direito Sanit, 2016; 17(2): 13-29.

16. SOUZA ECF, et. al. Acesso e acolhimento na atenção básica: uma análise de percepção dos usuários e profissionais de saúde. Cad. Saúde Pública, 2008; 24(supl.1): 5100-5110.

17. STOPA SR, et al. Acesso e uso de serviços de saúde pela população brasileira. Pesquisa Nacional de Saúde, 2013. Rev. Saúde Pública, 2017; (supl.1): 3s.

18. TEIXEIRA E. As três metodologias: acadêmica, da ciência e da pesquisa. 9. ed. Petrópolis (RJ): Vozes, 2009.

19. TRAVASSOS C, MARTINS M. Uma revisão sobre os conceitos de acesso a utilização de serviços de saúde. Cad. Saúde Pública, 2004; 20 (supl. 2): s190-8.

20. VIEGAS APB, et al. Fatores que influenciam o acesso aos serviços de saúde na visão de profissionais e usuários de unidade básica de referência. Saúde Soc, 2015; 24(1): 100-112.

21. WORLD HEALTH ORGANIZATION. Global health risk: mortaly and burden of disease attributable to selected major risk. Geneva: WHO; 2009. 